\title{
LEFT ORDERABLE SURGERIES OF DOUBLE TWIST KNOTS II
}

\author{
VU THE KHOI, MASAKAZU TERAGAITO, AND ANH T. TRAN
}

\begin{abstract}
A slope $r$ is called a left orderable slope of a knot $K \subset S^{3}$ if the 3 manifold obtained by $r$-surgery along $K$ has left orderable fundamental group. Consider two-bridge knots $C(2 m, \pm 2 n)$ and $C(2 m+1,-2 n)$ in the Conway notation, where $m \geq 1$ and $n \geq 2$ are integers. By using continuous families of hyperbolic $\mathrm{SL}_{2}(\mathbb{R})$ representations of knot groups, it was shown in HTe1, Tr2 that any slope in $(-4 n, 4 m)$ (resp. $[0, \max \{4 m, 4 n\}))$ is a left orderable slope of $C(2 m, 2 n)$ (resp. $C(2 m,-2 n))$ and in Ga2 that any slope in $(-4 n, 0]$ is a left orderable slope of $C(2 m+1,-2 n)$. However, the proofs of these results are incomplete since the continuity of the families of representations was not proved. In this paper, we complete these proofs and moreover we show that any slope in $(-4 n, 4 m)$ is a left orderable slope of $C(2 m+1,-2 n)$ detected by hyperbolic $\mathrm{SL}_{2}(\mathbb{R})$-representations of the knot group.
\end{abstract}

\section{INTRODUCTION}

The study of left orderability of fundamental groups of 3-manifolds obtained by Dehn surgeries along knots is motivated by the L-space conjecture of Boyer, Gordon and Watson [BGW] which states that an irreducible rational homology 3-sphere is an L-space if and only if its fundamental group is not left orderable. Here a rational homology 3-sphere $Y$ is an L-space if its Heegaard Floer homology $\widehat{\mathrm{HF}}(Y)$ has rank equal to the order of $H_{1}(Y ; \mathbb{Z})$, and a non-trivial group $G$ is left orderable if it admits a total ordering $<$ such that $g<h$ implies $f g<f h$ for all elements $f, g, h$ in $G$.

Many hyperbolic 3-manifolds are obtained by Dehn surgeries along knots. A slope $r$ is called a left orderable slope of a knot $K \subset S^{3}$ if the 3-manifold obtained by $r$-surgery along $K$ has left orderable fundamental group. Consider two-bridge knots $C(2 m, \pm 2 n)$ and $C(2 m+1,-2 n)$ in the Conway notation, where $m \geq 1$ and $n \geq 2$ are integers. By using continuous families of hyperbolic $\mathrm{SL}_{2}(\mathbb{R})$-representations of knot groups, it was shown in [HTe1, Tr2] that any slope in $(-4 n, 4 m)$ (resp. $[0, \max \{4 m, 4 n\}))$ is a left orderable slope of $C(2 m, 2 n)$ (resp. $C(2 m,-2 n))$ and in Ga2 that any slope in $(-4 n, 0]$ is a left orderable slope of $C(2 m+1,-2 n)$. However, the proofs of these results are incomplete since the continuity of the families of representations was not proved. More precisely, [HTe1, Proposition 4.2], [Tr2, Lemma 2.1] and [Ga2, Proposition 4.2] proved the existence of families of $\mathrm{SL}_{2}(\mathbb{R})$-representations of the knot groups but did not prove

The first author has been partially supported by the Vietnam National Foundation for Science and Technology Development (NAFOSTED) under grant number 101.04-2015.20.

The second author has been partially supported by JSPS KAKENHI Grant Number JP16K05149.

The third author has been partially supported by a grant from the Simons Foundation (\#354595). 
the continuity of these families. In this paper, we complete these proofs in Proposition 4.1. Proposition 4.2 and Proposition 5.1 respectively. Moreover, we extend the range of left orderable slopes of $C(2 m+1,-2 n)$ detected by hyperbolic $\mathrm{SL}_{2}(\mathbb{R})$-representations of their knot groups.

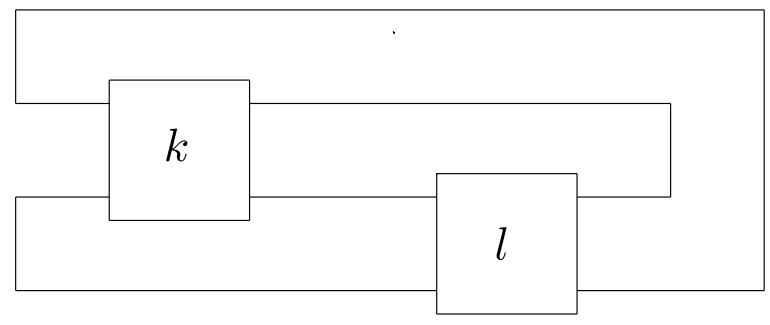

Figure 1. The two-bridge knot/link $C(k, l)$ in the Conway notation.

Theorem 1. Let $m \geq 1$ and $n \geq 2$ be integers. Then any slope in $(-4 n, 4 m)$ is a left orderable slope of $C(2 m+1,-2 n)$ detected by hyperbolic $\mathrm{SL}_{2}(\mathbb{R})$-representations of the knot group.

Remark 1.1. In [Tr1], by following the method of Culler-Dunfield's paper [CD] the third author used continuous families of elliptic $\mathrm{SL}_{2}(\mathbb{R})$-representations of knot groups to show that if $K$ is a two-bridge knot of the form $C(2 m,-2 n), C(2 m+1,2 n)$ or $C(2 m+1,-2 n)$, where $m \geq 1$ and $n \geq 1$ are integers, and

$$
\mathrm{LO}_{K}= \begin{cases}(-\infty, 1) & \text { if } K=C(2 m,-2 n) \\ (-\infty, 2 n-1) & \text { if } K=C(2 m+1,2 n) \\ (3-2 n, \infty) & \text { if } K=C(2 m+1,-2 n) \text { and } n \geq 2\end{cases}
$$

then any slope in $\mathrm{LO}_{K}$ is a left orderable slope of $K$.

Gao Ga2] independently showed that if $K$ is a two-bridge knot of the form $C(2 m+1,2 n)$ or $C(2 m+1,-2 n)$, where $m \geq 1$ and $n \geq 1$ are integers, and

$$
\mathrm{LO}_{K}^{\prime}= \begin{cases}(-\infty, 1) & \text { if } K=C(2 m+1,2 n), \\ (-1, \infty) & \text { if } K=C(2 m+1,-2 n) \text { and } n \geq 2,\end{cases}
$$

then any slope in $\mathrm{LO}_{K}^{\prime}$ is a left orderable slope of $K$. Her proof also used families of elliptic $\mathrm{SL}_{2}(\mathbb{R})$-representations of knot groups. Note that $\mathrm{LO}_{K}^{\prime}$ is a subset of $\mathrm{LO}_{K}$.

For more on the study of left orderable slopes of knots using hyperbolic $\mathrm{SL}_{2}(\mathbb{R})$ representations of knot groups, see [BGW, HTe2, Ga1].

This paper is organized as follows. In Section 2, we recall some facts about the universal covering group $\widehat{\mathrm{SL}_{2}(\mathbb{R})}$ of $\mathrm{SL}_{2}(\mathbb{R})$ and we study the lifting problem of a connected curve of hyperbolic $\mathrm{SL}_{2}(\mathbb{R})$-representations of knot groups. In Section 3, we review the Riley polynomial of two-bridge knots $C(k,-2 p)$, whose zero locus describes all non-abelian representations of the knot group into $\mathrm{SL}_{2}(\mathbb{C})$. In Section 3 we prove the existence of 
continuous families of hyperbolic $\mathrm{SL}_{2}(\mathbb{R})$-representations of the knot groups of $C(2 m, \pm 2 n)$ and therefore fix the gaps in [HTe1, Tr2]. In Section 4 we prove the existence of continuous families of hyperbolic $\mathrm{SL}_{2}(\mathbb{R})$-representations of the knot groups of $C(2 m+1,-2 n)$ and use it to give a proof of Theorem 1 .

\section{LifTING OF A CURVE OF HYPERBOLIC REPRESENTATIONS}

2.1. The group $\widetilde{\mathrm{SL}_{2}(\mathbb{R})}$. We recall some facts about the universal covering group $\widetilde{\mathrm{SL}_{2}(\mathbb{R})}$ (see [Kh] pages $763-764$ ). Let $\psi: \widetilde{\mathrm{SL}_{2}(\mathbb{R})} \rightarrow \mathrm{SL}_{2}(\mathbb{R})$ be the covering map. We can parameterize the universal covering group as

$$
\widetilde{\mathrm{SL}_{2}(\mathbb{R})}=\{(\gamma, \omega)|| \gamma \mid<1,-\infty<w<\infty\} .
$$

For an element $g=(\gamma, \omega) \in \widehat{\mathrm{SL}_{2}(\mathbb{R})}$, we will write $g[1]=\gamma$ and $g[2]=\omega$.

An element of $\mathrm{SL}_{2}(\mathbb{R})$ is called elliptic/parabolic/hyperbolic if it covers a matrix in $\mathrm{SL}_{2}(\mathbb{R})$ of the corresponding type.

The multiplication rule in the group $\widehat{\mathrm{SL}_{2}(\mathbb{R})}$ is given by $(\gamma, \omega)\left(\gamma^{\prime}, \omega^{\prime}\right)=\left(\gamma^{\prime \prime}, \omega^{\prime \prime}\right)$ where

$$
\begin{aligned}
\gamma^{\prime \prime} & =\frac{\gamma+\gamma^{\prime} e^{-2 i \omega}}{1+\bar{\gamma} \gamma^{\prime} e^{-2 i \omega}} \\
\omega^{\prime \prime} & =\omega+\omega^{\prime}+\arg \left(1+\bar{\gamma} \gamma^{\prime} e^{-2 i \omega}\right) .
\end{aligned}
$$

Let $A=\left(\begin{array}{ll}a & b \\ c & d\end{array}\right)$ be a matrix in $\mathrm{SL}_{2}(\mathbb{R})$ then

$$
\psi^{-1}(A)=\left\{\left(\frac{a-d+(b+c) i}{a+d+(b-c) i}, \arg (a+d+(b-c) i)+2 n \pi\right) \mid n \in \mathbb{Z}\right\} .
$$

Here, the function argument takes value in the interval $(-\pi, \pi]$. We note that if $\operatorname{Tr}(A)=$ $a+d>0$ then in the above formula we have $\arg (a+d+(b-c) i)=\arctan \left(\frac{b-c}{a+d}\right) \in\left(-\frac{\pi}{2}, \frac{\pi}{2}\right)$.

2.2. Lifting of a curve of hyperbolic representations. For a knot $K$ in $S^{3}$, let $X$ be an open tubular neighborhood of $K$ and let $G(K)=\pi_{1}(X)$ be the knot group of $K$ which is the fundamental group of $X$. Let $\mu$ be a meridian and $\lambda$ the canonical longitude. Recall that any representation $\rho: G(K) \rightarrow \mathrm{SL}_{2}(\mathbb{R})$ can be lifted to a representation $\tilde{\rho}$ into $\widehat{\mathrm{SL}_{2}(\mathbb{R})}$ because $H^{2}(K, \mathbb{Z})=0$. We know from $[\mathrm{Kh}$ that the lifts of $\rho$ come in a family. If we fix a lift $\widetilde{\rho}_{0}$, then we have a $\mathbb{Z}$-family of lifts $\widetilde{\rho}_{n}$ given by $\widetilde{\rho}_{n}(g)=\widetilde{\rho}_{0}(g) h_{n}(g)$, where $h_{n}$ is the representation

$$
\begin{array}{cccc}
G(K) & \longrightarrow & H_{1}(X) \equiv \mathbb{Z} & \longrightarrow \widehat{\mathrm{SL}_{2}(\mathbb{R})} \\
\mu & \longmapsto & 1 & \longmapsto(0, n \pi) .
\end{array}
$$

From this, we see that $\widetilde{\rho}_{n}(\lambda)$ does not depend on $n$.

An $\mathrm{SL}_{2}(\mathbb{R})$ representation $\rho$ of a knot group $G(K)$ is called hyperbolic if $\rho(\mu)$ and $\rho(\lambda)$ are hyperbolic elements. 
Recall that a hyperbolic element $g \in \widehat{\mathrm{SL}_{2}(\mathbb{R})}$ can be conjugated to a unique normal form $(\tanh (a), k \pi)$ if and only if $k \pi-\frac{\pi}{2}<g[2]<k \pi+\frac{\pi}{2}$. Let us fix an arbitrary lift $\widetilde{\rho}_{0}: G(K) \rightarrow \widetilde{\mathrm{SL}_{2}(\mathbb{R})}$ and suppose that $\widetilde{\rho}_{0}(\lambda)$ is conjugate to $(\tanh (b), k \pi)$. As noted above, the number $k$ does not depend on the chosen lift $\widetilde{\rho}_{0}$. We call $k$ the index of the representation $\rho$.

Lemma 2.1. Let $C$ be a connected curve of hyperbolic representations of a knot group $G(K)$ into $\mathrm{SL}_{2}(\mathbb{R})$. Then the indexes of all representations of $C$ are the same.

Proof. Note that as $\rho$ varies in $C$, the image $\widetilde{\rho}_{0}(\lambda)$ varies in a connected component of hyperbolic elements. Since each connected component corresponds to a single value of $k$ (see Figure 1 of $[\mathrm{Kh}]$ ), the number $k$ is the same for all representations in $C$.

We will also say that the curve $C$ in the above lemma has index $k$.

Corollary 2.2. Let $C$ be a connected curve of hyperbolic representations of a knot group $G(K)$ into $\mathrm{SL}_{2}(\mathbb{R})$. If $C$ contains a reducible representation then it has index 0.

Proof. Suppose that $\rho_{1} \in C$ is a reducible hyperbolic representation given by

$$
\rho_{1}\left(\mu_{i}\right)=\left(\begin{array}{cc}
s & a_{i} \\
0 & s^{-1}
\end{array}\right)
$$

where $\mu_{i}$ are generators of $G(K)$ which are conjugate to the standard meridian. Then we can connect $\rho_{1}$ to an abelian representation by using the curve

$$
\rho_{t}\left(\mu_{i}\right)=\left(\begin{array}{cc}
s & t a_{i} \\
0 & s^{-1}
\end{array}\right), t \in \mathbb{R} .
$$

It is easy to verify that if $\rho_{1}$ is a representation then so is $\rho_{t}$ for all $t$. As the abelian representation $\rho_{0}$ has index 0 , the corollary follows from the previous lemma.

The next proposition tells us how to find left orderable slopes of a knot, given a connected curve of hyperbolic representations of the knot group into $\mathrm{SL}_{2}(\mathbb{R})$.

Proposition 2.3. (1) Let $\left\{\rho_{y}: G(K) \rightarrow \mathrm{SL}_{2}(\mathbb{R})\right\}_{y}$ be a connected curve of hyperbolic representations of index 0 , and choose a lift $\widetilde{\rho}_{y}: G(K) \rightarrow \widehat{\mathrm{SL}_{2}(\mathbb{R})}$ such that $\tilde{\rho}_{y}(\mu)=(\tanh a(y), 0)$ and $\widetilde{\rho}_{y}(\lambda)=(\tanh b(y), 0)$. If $p / q$ is a slope such that $p / q=-b(y) / a(y)$ for some $y$, then $p / q$ is a left orderable slope of $K$.

(2) Let $\left\{\rho_{y}: G(K) \rightarrow \mathrm{SL}_{2}(\mathbb{R})\right\}_{y}$ be a connected curve of hyperbolic representations of index $k \neq 0$, and choose a lift $\widetilde{\rho}_{y}: G(K) \rightarrow \widetilde{\mathrm{SL}_{2}(\mathbb{R})}$ such that $\widetilde{\rho}_{y}(\mu)=(\tanh a(y), 0)$ and $\widetilde{\rho}_{y}(\lambda)=(\tanh b(y), k \pi)$. If $p / q$ is a slope such that $p / q=-b(y) / a(y)$ for some $y$ and $p \mid k$, then $p / q$ is a left orderable slope of $K$.

Proof. Let $X_{p / q}$ denote by 3-manifold obtained from $S^{3}$ by $p / q$-surgery along $K$. 
(1) Since $\widetilde{\rho}_{y}\left(\mu^{p} \lambda^{q}\right)=(\tanh (p a(y)+q b(y)), 0)=(0,0), \widetilde{\rho}_{y}$ gives a representation from $\pi_{1}\left(X_{p / q}\right)$ to $\widehat{\mathrm{SL}_{2}(\mathbb{R})}$. Note that $X_{p / q}$ is an irreducible 3-manifold (by $[\mathrm{HTh}]$ ) and $\widetilde{\mathrm{SL}_{2}(\mathbb{R})}$ is a left orderable group (by [Be]). Hence, by Theorem 1.1 of [BRW], $\pi_{1}\left(X_{p / q}\right)$ is a left orderable group. This means that $p / q$ is a left orderable slope of $K$.

(2) We choose another lift $\widetilde{\rho_{y}{ }^{\prime}}: G(K) \rightarrow \widehat{\mathrm{SL}_{2}(\mathbb{R})}$ such that $\tilde{\rho}_{y}^{\prime}(\mu)=\left(\tanh a(y),-\frac{k q}{p} \pi\right)$ and ${\widetilde{\rho_{y}}}^{\prime}(\lambda)=(\tanh b(y), k \pi)$. We then have

$$
{\tilde{\rho_{y}}}^{\prime}\left(\mu^{p} \lambda^{q}\right)=(\tanh (p a(y)+q b(y)),-k q \pi+k q \pi)=(0,0) .
$$

Therefore $\widetilde{\rho_{y}^{\prime}}$ gives a representation from $\pi_{1}\left(X_{p / q}\right)$ to $\widetilde{\mathrm{SL}_{2}(\mathbb{R})}$ and, as in $(1)$, the assertion follows.

\section{Representations of DOUble tWist KNOtS}

Consider the two-bridge knot/link $C(k, l)$ in the Conway notation, where $k, l$ are integers such that $|k l| \geq 3$. Note that $C(k, l)$ is the rational knot/link corresponding to continued fraction $k+1 / l$. It is easy to see that $C(k, l)$ is the mirror image of $C(l, k)=C(-k,-l)$. Moreover, $C(k, l)$ is a knot if $k l$ is even and is a two-component link if $k l$ is odd. In this paper, we only consider knots and so we can assume that $k>0$ and $l=-2 p$ is even.

Note that $C(k,-2 p)$ is the mirror image of the double twist knot $J(k, 2 p)$ in [HS]. Then, by [HS], the knot group of $C(k,-2 p)$ has a presentation

$$
G(C(k,-2 p))=\left\langle a, b \mid a w^{p}=w^{p} b\right\rangle
$$

where $a, b$ are meridians and

$$
w= \begin{cases}\left(a b^{-1}\right)^{m}\left(a^{-1} b\right)^{m} & \text { if } k=2 m, \\ \left(a b^{-1}\right)^{m} a b\left(a^{-1} b\right)^{m} & \text { if } k=2 m+1 .\end{cases}
$$

Moreover, the canonical longitude of $C(k,-2 p)$ corresponding to the meridian $\mu=a$ is $\lambda=\left(w^{p}\left(w^{p}\right)^{*} a^{-2 \varepsilon}\right)^{-1}$, where $\varepsilon=0$ if $k=2 m$ and $\varepsilon=2 p$ if $k=2 m+1$. Here, for a word $u$ in the letters $a, b$ we let $u^{*}$ be the word obtained by reading $u$ backwards.

Suppose $\rho: G(C(k,-2 p)) \rightarrow \mathrm{SL}_{2}(\mathbb{C})$ is a nonabelian representation. Up to conjugation, we may assume that

$$
\rho(a)=\left[\begin{array}{cc}
M & 1 \\
0 & M^{-1}
\end{array}\right] \text { and } \rho(b)=\left[\begin{array}{cc}
M & 0 \\
2-y & M^{-1}
\end{array}\right]
$$

where $(M, y) \in \mathbb{C}^{2}$ satisfies the matrix equation $\rho\left(a w^{p}\right)=\rho\left(w^{p} b\right)$. It is known that this matrix equation is equivalent to a single polynomial equation $R_{C(k,-2 p)}(x, y)=0$, where $x=(\operatorname{tr} \rho(a))^{2}$ and $R_{K}(x, y)$ is the Riley polynomial of a two-bridge knot $K$, see [Ri]. This polynomial can be described via the Chebychev polynomials as follows.

Let $\left\{S_{j}(v)\right\}_{j \in \mathbb{Z}}$ be the Chebychev polynomials in the variable $v$ defined by $S_{0}(v)=1$, $S_{1}(v)=v$ and $S_{j}(v)=v S_{j-1}(v)-S_{j-2}(v)$ for all integers $j$. Note that $S_{j}(v)=-S_{-j-2}(v)$ and $S_{j}( \pm 2)=( \pm 1)^{j}(j+1)$. Moreover, we see that $S_{j}(v)=\left(s^{j+1}-s^{-(j+1)}\right) /\left(s-s^{-1}\right)$ for 
$v=s+s^{-1} \neq \pm 2$ from the recurrence relation. Using these identities one can prove the following.

Lemma 3.1. We have

(1) $S_{j}^{2}(v)-v S_{j}(v) S_{j-1}(v)+S_{j-1}^{2}(v)=1$ for any integer $j$,

(2) $S_{n}(v)=\prod_{j=1}^{n}\left(v-2 \cos \frac{j \pi}{n+1}\right)$ for any positive integer $n$,

(3) $S_{n}(v)-S_{n-1}(v)=\prod_{j=1}^{n}\left(v-2 \cos \frac{(2 j-1) \pi}{2 n+1}\right)$ for any positive integer $n$.

Proof. (1) By the recurrence relation,

$$
\begin{aligned}
S_{j}^{2}(v)-v S_{j}(v) S_{j-1}+S_{j-1}^{2}(v) & =S_{j}(v)\left(S_{j}(v)-v S_{j-1}(v)\right)+S_{j-1}^{2}(v) \\
& =\left(v S_{j-1}(v)-S_{j-2}(v)\right)\left(-S_{j-2}(v)\right)+S_{j-1}^{2}(v) \\
& =S_{j-1}^{2}(v)-v S_{j-1}(v) S_{j-2}(v)+S_{j-2}^{2}(v) .
\end{aligned}
$$

Since $S_{1}(v)^{2}-v S_{1}(v) S_{0}(v)+S_{0}^{2}(v)=1$, we have the conclusion.

(2) For any positive integer $n, S_{n}(v)$ is a polynomial of degree $n$. Since \pm 2 are not roots of $S_{n}(v)$, all roots come from solving $s^{n+1}-s^{-(n+1)}=0$, where $v=s+s^{-1}$. Hence, the conclusion follows from the observation that $2 \cos j \pi /(n+1)(j=1,2, \ldots, n)$ give all roots of $S_{n}(v)$.

(3) For any positive integer $n, S_{n}(v)-S_{n-1}(v)$ is a polynomial of degree $n$, and

$$
\begin{aligned}
S_{n}(v)-S_{n-1}(v) & =\frac{s^{n+1}-s^{-(n+1)}}{s-s^{-1}}-\frac{s^{n}-s^{-n}}{s-s^{-1}} \\
& =\frac{s^{n+1}-s^{-(n+1)}-s^{n}+s^{-n}}{s-s^{-1}} \\
& =\frac{s^{-(n+1)}}{s-s^{-1}} \cdot\left(s^{2 n+2}-1-s^{2 n+1}+s\right) \\
& =\frac{s^{-(n+1)}}{s-s^{-1}}(s-1)\left(s^{2 n+1}+1\right) .
\end{aligned}
$$

Hence all roots of $S_{n}(v)-S_{n-1}(v)$ come from solving $s^{2 n+1}+1=0$. That is, $2 \cos (2 j-$ $1) \pi /(2 n+1)(j=1,2, \ldots, n)$ give all the roots.

The Riley polynomial of $C(k,-2 p)$, whose zero locus describes all non-abelian representations of the knot group of $C(k,-2 p)$ into $\mathrm{SL}_{2}(\mathbb{C})$, is

$$
R_{C(k,-2 p)}(x, y)=S_{p}(t)-z S_{p-1}(t)
$$

where

$$
t=\operatorname{tr} \rho(w)= \begin{cases}2+(y+2-x)(y-2) S_{m-1}^{2}(y) & \text { if } k=2 m, \\ 2-(y+2-x)\left(S_{m}(y)-S_{m-1}(y)\right)^{2} & \text { if } k=2 m+1,\end{cases}
$$

and

$$
z= \begin{cases}1+(y+2-x) S_{m-1}(y)\left(S_{m}(y)-S_{m-1}(y)\right) & \text { if } k=2 m, \\ 1-(y+2-x) S_{m}(y)\left(S_{m}(y)-S_{m-1}(y)\right) & \text { if } k=2 m+1 .\end{cases}
$$


Moreover, for the representation $\rho: G(C(k,-2 p)) \rightarrow \mathrm{SL}_{2}(\mathbb{C})$ of the form (3.1) the image of the canonical longitude $\lambda=\left(w^{p}\left(w^{p}\right)^{*} a^{-2 \varepsilon}\right)^{-1}$ has the form $\rho(\lambda)=\left[\begin{array}{cc}L & * \\ 0 & L^{-1}\end{array}\right]$, where

$$
L=-\frac{M^{-1}\left(S_{m}(y)-S_{m-1}(y)\right)-M\left(S_{m-1}(y)-S_{m-2}(y)\right)}{M\left(S_{m}(y)-S_{m-1}(y)\right)-M^{-1}\left(S_{m-1}(y)-S_{m-2}(y)\right)} \quad \text { if } k=2 m
$$

and

$$
L=-M^{4 p} \frac{M^{-1} S_{m}(y)-M S_{m-1}(y)}{M S_{m}(y)-M^{-1} S_{m-1}(y)} \quad \text { if } k=2 m+1 .
$$

See e.g. [Tr2, $\mathrm{Pe}]$.

\section{The CASE of $C(2 m, \pm 2 n)$}

In this section we prove the existence of continuous families of hyperbolic $\mathrm{SL}_{2}(\mathbb{R})$ representations of knot groups of $C(2 m, \pm 2 n)$ and hence fix the gaps in [HTe1, Tr2].

Proposition 4.1. There exist $n-1$ continuous real functions $x_{j}:(2, \infty) \rightarrow(0, \infty)$, where $1 \leq j \leq n-1$, in the variable $y$ such that $R_{C(2 m, 2 n)}\left(x_{j}(y), y\right)=0$ and

$$
y+2+\frac{4 \sin ^{2} \frac{(2 j-1) \pi}{4 n+2}}{(y-2) S_{m-1}^{2}(y)}<x_{j}(y)<y+2+\frac{4 \sin ^{2} \frac{(2 j+1) \pi}{4 n+2}}{(y-2) S_{m-1}^{2}(y)}
$$

for all $y>2$.

Proof. Let $K=C(2 m, 2 n)$. We have $R_{K}(x, y)=S_{-n}(t)-z S_{-n-1}(t)$ where

$$
\begin{aligned}
t & =2+(y+2-x)(y-2) S_{m-1}^{2}(y) \\
z & =1+(y+2-x) S_{m-1}(y)\left(S_{m}(y)-S_{m-1}(y)\right) .
\end{aligned}
$$

Note that $R_{K}(x, y)=(t-z) S_{-n-1}(t)-S_{-n-2}(t)=S_{n}(t)-(t-z) S_{n-1}(t)$.

Let $t_{j}=2 \cos \frac{(2 j-1) \pi}{2 n+1}$ for $j=1, \ldots, n$. Then, Lemma 3.1(3) gives $S_{n}(t)-S_{n-1}(t)=$ $\prod_{j=1}^{n}\left(t-t_{j}\right)$, and the signs of $S_{n}\left(t_{j}\right)$ change alternately as $S_{n}\left(t_{1}\right)>0, S_{n}\left(t_{2}\right)<0, \ldots$, because of the inequality

$$
\frac{j-1}{n+1}<\frac{2 j-1}{2 n+1}<\frac{j}{n+1}(j=1,2, \ldots, n)
$$

and Lemma 3.1(2).

Fix a real number $y>2$. Let $s_{j}(y)=y+2+\frac{2-t_{j}}{(y-2) S_{m-1}^{2}(y)}$ for $j=1, \ldots, n$. Since $-2<t_{n}<\cdots<t_{1}<2$, we have $s_{n}(y)>\cdots>s_{1}(y)>y+2$. At $x=s_{j}(y)$ we have $t=t_{j}$ and so $S_{n}(t)=S_{n-1}(t)$. This implies that

$$
\begin{aligned}
R_{K}\left(s_{j}(y), y\right) & =(1-(t-z)) S_{n}\left(t_{j}\right) \\
& =\left(y+2-s_{j}(y)\right) S_{m-1}(y)\left(S_{m-1}(y)-S_{m-2}(y)\right) S_{n}\left(t_{j}\right) \\
& =-\frac{2-t_{j}}{(y-2) S_{m-1}(y)}\left(S_{m-1}(y)-S_{m-2}(y)\right) S_{n}\left(t_{j}\right) .
\end{aligned}
$$


Since $y>2$, we have $S_{m-1}(y)-S_{m-2}(y)>0$ and $S_{m-1}(y)>0$ by Lemma 3.1. Hence $R_{K}\left(s_{j}(y), y\right)$ and $S_{n}\left(t_{j}\right)$ have opposite signs, so the sign of $R_{K}\left(s_{j}(y), y\right)$ changes alternately as $R_{K}\left(s_{1}(y), y\right)<0, R_{K}\left(s_{2}(y), y\right)>0, \ldots$

For each $1 \leq j \leq n-1$, since $R_{K}\left(s_{j}(y), y\right) R_{K}\left(s_{j+1}(y), y\right)<0$, there exists $x_{j}(y) \in$ $\left(s_{j}(y), s_{j+1}(y)\right)$ such that $R_{K}\left(x_{j}(y), y\right)=0$. Also, since $R_{K}(y+2, y)=1$ and $R_{K}\left(s_{1}(y), y\right)<$ 0 , there exists $x_{0}(y) \in\left(y+2, s_{1}(y)\right)$ such that $R_{K}\left(x_{0}(y), y\right)=0$.

Since $R_{K}(x, y)=S_{n}(t)-(t-z) S_{n-1}(t)=z S_{n-1}(t)-S_{n-2}(t)$, we see that $R_{K}(x, y)$ is a polynomial of degree $n$ in $x$ for each fixed real number $y>2$. This polyomial has exactly $n$ simple real roots $x_{0}(y), \ldots, x_{n-1}(y)$ satisfying $x_{n-1}(y)>\cdots>x_{0}(y)>y+2$, hence the implicit function theorem implies that each $x_{j}(y)$ is a continuous function in $y>2$. The continuous functions $x_{1}(y), \ldots, x_{n-1}(y)$ satisfy the conditions of Proposition 4.1.

Proposition 4.2. There exist $n-1$ continuous real functions $x_{j}:(2, \infty) \rightarrow(0, \infty)$, where $1 \leq j \leq n-1$, in the variable $y$ such that $R_{C(2 m,-2 n)}\left(x_{j}(y), y\right)=0$ and

$$
y+2+\frac{4 \sin ^{2} \frac{(2 j-1) \pi}{4 n+2}}{(y-2) S_{m-1}^{2}(y)}<x_{j}(y)<y+2+\frac{4 \sin ^{2} \frac{(2 j+1) \pi}{4 n+2}}{(y-2) S_{m-1}^{2}(y)}
$$

for all $y>2$.

Proof. Let $K=C(2 m,-2 n)$. We have $R_{K}(x, y)=S_{n}(t)-z S_{n-1}(t)$ where

$$
\begin{aligned}
t & =2+(y+2-x)(y-2) S_{m-1}^{2}(y), \\
z & =1+(y+2-x) S_{m-1}(y)\left(S_{m}(y)-S_{m-1}(y)\right) .
\end{aligned}
$$

Fix a real number $y \geq 2$. Choose $t_{j}$ and $s_{j}(y)$ for $1 \leq j \leq n$ as in the proof of Proposition 4.1. Recall $S_{n}(t)=S_{n-1}(t)$ at $t=t_{j}$. Since

$$
\begin{aligned}
R_{K}\left(s_{j}(y), y\right) & =(1-z) S_{n}\left(t_{j}\right) \\
& =-\left(y+2-s_{j}(y)\right) S_{m-1}(y)\left(S_{m}(y)-S_{m-1}(y)\right) S_{n}\left(t_{j}\right) \\
& =\frac{2-t_{j}}{(y-2) S_{m-1}(y)}\left(S_{m}(y)-S_{m-1}(y)\right) S_{n}\left(t_{j}\right),
\end{aligned}
$$

$R_{K}\left(s_{j}(y), y\right)$ and $S_{n}\left(t_{j}\right)$ have the same sign. So, the sign of $R_{K}\left(s_{j}(y), y\right)$ changes alternately. Since $R_{K}\left(s_{j}(y), y\right) R_{K}\left(s_{j+1}(y), y\right)<0$, there exists $x_{j}(y) \in\left(s_{j}(y), s_{j+1}(y)\right)$ such that $R_{K}\left(x_{j}(y), y\right)=0$ for each $1 \leq j \leq n-1$.

We now claim that there exists $x_{0}(y) \in(0, y+2)$ such that $R_{K}\left(x_{0}(y), y\right)=0$. Indeed, at $x=0$ we have $t=2+\left(y^{2}-4\right) S_{m-1}^{2}(y)$ and $z=1+(y+2) S_{m-1}(y)\left(S_{m}(y)-S_{m-1}(y)\right)$. Write $y=\xi+\xi^{-1}$ for some $\xi>1$. Then $S_{k}(y)=\frac{\xi^{k+1}-\xi^{-(k+1)}}{\xi-\xi^{-1}}$ for all integers $k$.

Claim 4.3. (1) $t=\xi^{2 m}+\xi^{-2 m}$.

(2) $z=\xi^{-2 m} \frac{\xi^{4 m+1}-1}{\xi-1}$.

Proof of Claim 4.3. (1) follows from

$$
t=2+\left(y^{2}-4\right) S_{m-1}^{2}(y)
$$




$$
\begin{aligned}
& =2+\left(\xi-\xi^{-1}\right)^{2}\left(\frac{\xi^{m}-\xi^{-m}}{\xi-\xi^{-1}}\right)^{2} \\
& =2+\left(\xi^{m}-\xi^{-m}\right)^{2} \\
& =\xi^{2 m}+\xi^{-2 m} .
\end{aligned}
$$

(2) Similarly,

$$
\begin{aligned}
z & =1+\left(\xi+\xi^{-1}+2\right) \frac{\xi^{m}-\xi^{-m}}{\xi-\xi^{-1}}\left(\frac{\xi^{m+1}-\xi^{-(m+1)}}{\xi-\xi^{-1}}-\frac{\xi^{m}-\xi^{-m}}{\xi-\xi^{-1}}\right) \\
& =\frac{1}{\left(\xi-\xi^{-1}\right)^{2}}\left(1+\left(\xi+\xi^{-1}+2\right)\left(\xi^{m}-\xi^{-m}\right)\left(\xi^{m+1}-\xi^{-(m+1)}-\xi^{m}+\xi^{-m}\right)\right) \\
& =\frac{1}{\left(\xi-\xi^{-1}\right)^{2}}\left(\xi^{2 m+2}+\xi^{-(2 m+2)}+\xi^{2 m+1}+\xi^{-(2 m+1)}-\xi^{2 m}-\xi^{-2 m}-\xi^{2 m-1}-\xi^{-(2 m-1)}\right) \\
& =\frac{\xi^{2}-1}{\left(\xi-\xi^{-1}\right)^{2}}\left(\xi^{2 m}+\xi^{2 m-1}-\xi^{-(2 m+1)}-\xi^{-(2 m+2)}\right) \\
& =\frac{\xi^{2}}{\xi^{2}-1} \xi^{-(2 m+2)}\left(\xi^{4 m+2}+\xi^{4 m+1}-\xi-1\right) \\
& =\xi^{-2 m} \frac{\xi^{4 m+1}-1}{\xi-1} .
\end{aligned}
$$

By Claim 4.3 $(1), S_{k}(t)=\frac{\xi^{2 m(k+1)}-\xi^{-2 m(k+1)}}{\xi^{2 m}-\xi^{-2 m}}$ for all integers $k$. Hence we have

$$
\begin{aligned}
& R_{K}(0, y)=S_{n}(t)-z S_{n-1}(t) \\
& =\frac{\xi^{2 m(n+1)}-\xi^{-2 m(n+1)}}{\xi^{2 m}-\xi^{-2 m}}-\xi^{-2 m} \frac{\xi^{4 m+1}-1}{\xi-1} \frac{\xi^{2 m n}-\xi^{-2 m n}}{\xi^{2 m}-\xi^{-2 m}} \\
& =\frac{1}{\xi^{2 m}-\xi^{-2 m}}\left(\left(\xi^{2 m(n+1)}-\xi^{-2 m(n+1)}\right)-\xi^{-2 m} \frac{\xi^{4 m+1}-1}{\xi-1}\left(\xi^{2 m n}-\xi^{-2 m n}\right)\right) \\
& =\frac{\xi^{-2 m n}}{\xi^{2 m}-\xi^{-2 m}}\left(\left(\xi^{2 m(2 n+1)}-\xi^{-2 m}\right)-\xi^{-2 m} \frac{\xi^{4 m+1}-1}{\xi-1}\left(\xi^{4 m n}-1\right)\right) \\
& =\frac{\xi^{-2 m n}}{\xi^{2 m}-\xi^{-2 m}} \frac{1}{\xi-1}\left(\xi^{4 m n+2 m}-\xi^{4 m n-2 m}-\xi^{2 m+1}+\xi^{-(2 m-1)}\right) \\
& =-\xi^{-2 m n} \frac{\xi^{4 m n}-\xi}{\xi-1}<0 \text {. }
\end{aligned}
$$

Since $R_{K}(y+2, y)=1$, there exists $x_{0}(y) \in(0, y+2)$ such that $R_{K}\left(x_{0}(y), y\right)=0$.

By writing $R_{K}(x, y)=S_{n}(t)-z S_{n-1}(t)=(t-z) S_{n-1}(t)-S_{n-2}(t)$ and noting that

$$
t-z=1+(y+2-x) S_{m-1}(y)\left(-S_{m-1}(y)+S_{m-2}(y)\right),
$$

we see that $R_{K}(x, y)$ is a polynomial of degree $n$ in $x$ for a fixed real number $y>2$. This polynomial has exactly $n$ simple real roots $x_{0}(y), \ldots, x_{n-1}(y)$ satisfying $x_{n-1}(y)>\cdots>$ $x_{1}(y)>y+2>x_{0}(y)>0$, hence the implicit function theorem implies that each $x_{j}(y)$ 
is a continuous function in $y>2$. The continuous functions $x_{1}(y), \ldots, x_{n-1}(y)$ satisfy the conditions of Proposition 4.2.

\section{THE CASE OF $C(2 m+1,-2 n)$}

In this section we prove the existence of continuous families of hyperbolic $\mathrm{SL}_{2}(\mathbb{R})$ representations of the knot groups of $C(2 m+1,-2 n)$ and use it to give a proof of Theorem 1.

\subsection{Real roots of the Riley polynomial.}

Proposition 5.1. There exists a unique continuous real function $x:\left(2 \cos \frac{\pi}{2 m+1}, \infty\right) \rightarrow$ $(0, \infty)$ in the variable $y$ such that $R_{C(2 m+1,-2 n)}(x(y), y)=0$ and $x(y)>y+2$ for all $y>2 \cos \frac{\pi}{2 m+1}$.

Proof. Let $K=C(2 m+1,-2 n)$. We have $R_{K}(x, y)=S_{n}(t)-z S_{n-1}(t)$ where

$$
\begin{aligned}
t & =2-(y+2-x)\left(S_{m}(y)-S_{m-1}(y)\right)^{2}, \\
z & =1-(y+2-x) S_{m}(y)\left(S_{m}(y)-S_{m-1}(y)\right) .
\end{aligned}
$$

Choose $t_{j}$ for $1 \leq j \leq n$ as in the proof of Proposition 4.1. Fix a real number $y>2 \cos \frac{\pi}{2 m+1}$. Then $S_{m}(y)>S_{m-1}(y)>0$ by Lemma 3.1(3). Let $s_{j}(y)=y+2-$ $\frac{2-t_{j}}{\left(S_{m}(y)-S_{m-1}(y)\right)^{2}}$ for $j=1, \ldots, n$. Then $s_{n}(y)<\cdots<s_{1}(y)<y+2$. Since

$$
R_{K}\left(s_{j}(y), y\right)=(1-z) S_{n}\left(t_{j}\right)=\left(y+2-s_{j}(y)\right) S_{m}(y)\left(S_{m}(y)-S_{m-1}(y)\right) S_{n}\left(t_{j}\right),
$$

$R_{K}\left(s_{j}(y), y\right)$ and $S_{n}\left(t_{j}\right)$ have the same sign. Thus the sign of $R_{K}\left(s_{j}(y), y\right)$ changes alternately. Hence, there exists $x_{j}(y) \in\left(s_{j+1}(y), s_{j}(y)\right)$ such that $R_{K}\left(x_{j}(y), y\right)=0$ for each $1 \leq j \leq n-1$.

By writing $R_{K}(x, y)=(t-z) S_{n-1}(t)-S_{n-2}(t)$ and noting that

$$
t-z=1+(y+2-x)\left(S_{m}(y)-S_{m-1}(y)\right) S_{m-1}(y),
$$

we see that $R_{K}(x, y)$ is a polynomial of degree $n$ in $x$ with negative highest coefficient for each fixed real number $y>2 \cos \frac{\pi}{2 m+1}$. For, $t$ (resp. $t-z$ ) has degree one in the variable $x$ with positive (resp. negative) coefficient, and the Chebyshev polynomials $S_{n-1}(t)$ and $S_{n-2}(t)$ are polynomials of degree $n-1$ and $n-2$, respectively, in $t$ with positive highest coefficient. Since $\lim _{x \rightarrow \infty} R_{K}(x, y)=-\infty$ and $R_{K}(y+2, y)=1$, there exists $x_{0}(y) \in(y+2, \infty)$ such that $R_{K}\left(x_{0}(y), y\right)=0$. For a fixed real number $y>2 \cos \frac{\pi}{2 m+1}$, the polynomial $R_{K}(x, y)$ of degree $n$ in $x$ has exactly $n$ simple real roots $x_{0}(y), \ldots, x_{n-1}(y)$ satisfying $x_{n-1}(y)<\cdots<x_{1}(y)<y+2<x_{0}(y)$, hence the implicit function theorem implies that each $x_{j}(y)$ is a continuous function in $y>2 \cos \frac{\pi}{2 m+1}$.

Letting $x(y)=x_{0}(y)$ for $y>2 \cos \frac{\pi}{2 m+1}$, we have $x(y)>y+2$ and $R_{K}(x(y), y)=0$.

Proposition 5.2. The continuous real function $x(y)$ in Proposition 5.1 satisfies the following properties: 
(1) $x(y)>2+\frac{S_{m}(y)}{S_{m-1}(y)}+\frac{S_{m-1}(y)}{S_{m}(y)}>4$ for all $y>2 \cos \frac{\pi}{2 m+1}$,

(2) $x(y) \rightarrow \infty$ as $y \rightarrow\left(2 \cos \frac{\pi}{2 m+1}\right)^{+}$,

(3) $y^{2 m+2 n-2}\left(x(y)-2-\frac{S_{m}(y)}{S_{m-1}(y)}-\frac{S_{m-1}(y)}{S_{m}(y)}\right) \rightarrow 1$ as $y \rightarrow \infty$.

Proof. (1) Since $R_{K}(x(y), y)=0$ we have $S_{n}(t)=z S_{n-1}(t)$. By Lemma 3.1(1), $S_{n}^{2}(t)-$ $t S_{n}(t) S_{n-1}(t)+S_{n-1}^{2}(t)=1$. Thus we have $\left(z^{2}-t z+1\right) S_{n-1}^{2}(t)=1$. Let $G=S_{m}(y)$ and $H=S_{m-1}(y)$. Then $G>H>0$ for $y>2 \cos \frac{\pi}{2 m+1}$ by Lemma 3.1(3). By using $G^{2}-y G H+H^{2}=1$ and $t-2=(x-y-2)(G-H)^{2}$, we have

$$
\begin{aligned}
z^{2}-t z+1 & =(z-1)^{2}-(t-2) z \\
& =(x-y-2)^{2} G^{2}(G-H)^{2}-(x-y-2)(G-H)^{2}(1+(x-y-2) G(G-H)) \\
& =(x-y-2)(G-H)^{2}((x-y-2) G H-1) \\
& =(x-y-2)(G-H)^{2}\left((x-2) G H-G^{2}-H^{2}\right) \\
& =(t-2)\left((x-2) G H-G^{2}-H^{2}\right) .
\end{aligned}
$$

Hence $(t-2)\left((x-2) G H-G^{2}-H^{2}\right) S_{n-1}^{2}(t)=1$. Since $t-2=(x-y-2)(G-H)^{2}>0$, we get $(x-2) G H-G^{2}-H^{2}>0$. This implies that

$$
x>2+\frac{G^{2}+H^{2}}{G H}=4+\frac{(G-H)^{2}}{G H}>4 .
$$

(2) As $y \rightarrow\left(2 \cos \frac{\pi}{2 m+1}\right)^{+}$we have $G-H \rightarrow 0$ by Lemma 3.1(3). If $x$ is bounded, then $t-2=(x-2-y)(G-H)^{2} \rightarrow 0$ and $1=(t-2)\left((x-2) G H-G^{2}-H^{2}\right) S_{n-1}^{2}(t) \rightarrow 0$, a contradiction. Hence $x(y) \rightarrow \infty$ as $y \rightarrow\left(2 \cos \frac{\pi}{2 m+1}\right)^{+}$.

(3) We now consider the case $y>2$. First,

$$
\begin{aligned}
t+2-x & =(x-y-2)(G-H)^{2}+4-x \\
& =(x-y-2)(1+(y-2) G H)+4-x \\
& =(x-y-2)(y-2) G H-y+2 \\
& =(y-2)((x-y-2) G H-1) \\
& =(y-2)\left((x-2) G H-G^{2}-H^{2}\right)>0 .
\end{aligned}
$$

Hence $t>x-2>y$. Noting that $(t-2)\left((x-2) G H-G^{2}-H^{2}\right) S_{n-1}^{2}(t)=1$, we have $(x-2) G H-G^{2}-H^{2} \rightarrow 0$ as $y \rightarrow \infty$. This is equivalent to $G H(x-2-y)-1=$ $G H \frac{t-2}{(G-H)^{2}}-1 \rightarrow 0$ as $y \rightarrow \infty$. Since

$$
G H \frac{t-2}{(G-H)^{2}}=\frac{\frac{t}{y}-\frac{2}{y}}{1-\frac{2}{y}+\frac{1}{y G H}},
$$

$\frac{t}{y} \rightarrow 1$ as $y \rightarrow \infty$. The equation $(t-2)\left((x-2) G H-G^{2}-H^{2}\right) S_{n-1}^{2}(t)=1$ gives

$$
(t-2) G H\left(x-2-\frac{G}{H}-\frac{H}{G}\right) S_{n-1}^{2}(t)=1 .
$$


Since $G$ and $H$ have degree $m$ and $m-1$ respectively in $y$ with positive highest coefficient and $S_{n-1}(t)^{2}$ has degree $2 n-2$ in $t, y^{2 m+2 n-2}\left(x-2-\frac{G}{H}-\frac{H}{G}\right) \rightarrow 1$ as $y \rightarrow \infty$. This completes the proof of Proposition 5.2.

5.2. Proof of Theorem 1. Let $X$ be the complement of an open tubular neighborhood of $K=C(2 m+1,-2 n)$ in $S^{3}$, and $X_{r}$ the 3 -manifold obtained from $S^{3}$ by $r$-surgery along $K$. Consider the function $x(y)$ in Proposition 5.1. For each $y>2 \cos \frac{\pi}{2 m+1}$, we have $x(y)>2+\frac{S_{m}(y)}{S_{m-1}(y)}+\frac{S_{m-1}(y)}{S_{m}(y)}>4$ by Proposition 5.2(1). Let $M(y)=\frac{1}{2}(\sqrt{x(y)}+$ $\sqrt{x(y)-4})>1$, then $\sqrt{x(y)}=M(y)+M(y)^{-1}$. Since $R_{K}(x(y), y)=0$, there exists a non-abelian representation $\rho_{y}: \pi_{1}(X) \rightarrow \mathrm{SL}_{2}(\mathbb{R})$ such that

$$
\rho_{y}(a)=\left[\begin{array}{cc}
M(y) & 1 \\
0 & M(y)^{-1}
\end{array}\right] \text { and } \rho_{y}(b)=\left[\begin{array}{cc}
M(y) & 0 \\
2-y(x) & M(y)^{-1}
\end{array}\right] .
$$

Moreover, the image of the canonical longitude $\lambda$ corresponding to the meridian $\mu=a$ has the form $\rho_{y}(\lambda)=\left[\begin{array}{cc}L(y) & * \\ 0 & L(y)^{-1}\end{array}\right]$, where

$$
L(y)=-M(y)^{4 n} \frac{M(y)^{-1} S_{m}(y)-M(y) S_{m-1}(y)}{M(y) S_{m}(y)-M(y)^{-1} S_{m-1}(y)} .
$$

As in the proof of Proposition 5.2, we let $G=S_{m}(y)$ and $H=S_{m-1}(y)$. Then $G>H>$ 0 for $y>2 \cos \frac{\pi}{2 m+1}$ and $L(y)=M(y)^{4 n} \frac{M(y)^{2}-\frac{G}{H}}{M(y)^{2} \frac{G}{H}-1}$. Since $M(y)^{2}+M(y)^{-2}=x(y)-2>$ $\frac{G}{H}+\frac{H}{G}$, we have $M(y)^{2}>\frac{G}{H}>1$. This implies that

$$
L(y)=M(y)^{4 n} \frac{M(y)^{2}-\frac{G}{H}}{M(y)^{2} \frac{G}{H}-1}>0 .
$$

As $y \rightarrow\left(2 \cos \frac{\pi}{2 m+1}\right)^{+}$we have $\frac{G}{H}=\frac{S_{m}(y)}{S_{m-1}(y)} \rightarrow 1$ and $M(y) \rightarrow \infty$ (by Proposition $5.2(2))$, so $\frac{M(y)^{2}-\frac{G}{H}}{M(y)^{2} \frac{G}{H}-1} \rightarrow 1$. Hence

$$
\frac{\log L(y)}{\log M(y)}=4 n+\frac{\log \frac{M(y)^{2}-\frac{G}{H}}{M(y)^{2} \frac{G}{H}-1}}{\log M(y)} \rightarrow 4 n .
$$

As $y \rightarrow \infty$ we have $y^{2 m+2 n-2}\left(x(y)-2-\frac{G}{H}-\frac{H}{G}\right) \rightarrow 1$ by Proposition $5.2(3)$. This is equivalent to $y^{2 m+2 n-2}\left(M(y)^{2}-\frac{G}{H}\right)\left(1-\frac{1}{M(y)^{2} \frac{G}{H}}\right) \rightarrow 1$, which implies that $y^{2 m+2 n-2}\left(M(y)^{2}-\right.$ $\left.\frac{G}{H}\right) \rightarrow 1$. Thus $M(y)^{2}-\frac{G}{H} \rightarrow 0$. Since $G$ and $H$ have degree $m$ and $m-1$ respectively in $y$ with positive highest coefficient, $\frac{G}{H}-y \rightarrow 0$. Then $M(y)^{2}-y \rightarrow 0$. Asymptotically, $M(y)^{2}-\frac{G}{H} \sim y^{2-2 m-2 n}$ and $M(y)^{2} \frac{G}{H}-1 \sim y^{2}$. Hence

$$
\frac{\log L(y)}{\log M(y)}=4 n+\frac{\log \frac{M(y)^{2}-\frac{G}{H}}{M(y)^{2} \frac{G}{H}-1}}{\log M(y)} \rightarrow 4 n-(4 m+4 n)=-4 m .
$$

Consider the continuous function $f(y):=-\frac{\log L(y)}{\log M(y)}$ for $y>2 \cos \frac{\pi}{2 m+1}$. Then from the above arguments we conclude that the image of $f$ contains the interval $(-4 n, 4 m)$. 
This implies that for any slope $r \in(-4 n, 4 m)$ there exists $y>2 \cos \frac{\pi}{2 m+1}$ such that $r=f(y)=-\frac{\log L(y)}{\log M(y)}$.

The continuous family $C$ of nonabelian representations $\left\{\rho_{y}\right\}, y>2 \cos \frac{\pi}{2 m+1}$, contains a special one which is the reducible nonabelian representation $\rho_{2}$ (i.e. $\rho_{y}$ at $y=2$ ). This representation has index 0 by Corollary 2.2 and so by Lemma 2.1 the continuous family $C$ has index 0. Applying Proposition 2.3(1) to $C$, with $a(y)=\log M(y)$ and $b(y)=\log L(y)$, we conclude that any slope $r \in(-4 n, 4 m)$ is a left orderable slope of $K=C(2 m+1,-2 n)$ detected by hyperbolic $\mathrm{SL}_{2}(\mathbb{R})$-representations of the knot group.

\section{REFERENCES}

[Be] G. Bergman, Right orderable groups that are not locally indicable, Pacific J. Math. 147 (1991), no. $2,243-248$.

[BGW] S. Boyer, C. Gordon and L. Watson, On L-spaces and left-orderable fundamental groups, Math. Ann. 356 (2013), no. 4, 1213-1245.

[BRW] S. Boyer, D. Rolfsen and B. Wiest, Orderable 3-manifold groups, Ann. Inst. Fourier (Grenoble) 55 (2005), no. 1, 243-288.

[CD] M. Culler and N. Dunfield, Orderability and Dehn filling, Geom. Topol. 22 (2018), no. 3, 14051457.

[Ga1] X. Gao, Orderability of homology spheres obtained by Dehn filling, preprint, arXiv:1810.11202.

[Ga2] X. Gao, Slope of orderable Dehn filling of two-bridge knots, preprint, arXiv:1912.07468.

[HS] J. Hoste and P. Shanahan, A formula for the A-polynomial of twist knots, J. Knot Theory Ramifications 13 (2004), no. 2, 193-209.

[HTe1] R. Hakamata and M. Teragaito, Left-orderable fundamental groups and Dehn surgery on genus one 2-bridge knots, Algebr. Geom. Topol. 14 (2014), no. 4, 2125-2148.

[HTe2] R. Hakamata and M. Teragaito, Left-orderable fundamental group and Dehn surgery on the knot $5_{2}$, Canad. Math. Bull. 57 (2014), no. 2, 310-317.

[HTh] A. Hatcher and W. Thurston, Incompressible surfaces in 2-bridge knot complements, Invent. Math. 79 (1985), no. 2, 225-246.

[Kh] V. Khoi, A cut-and-paste method for computing the Seifert volumes, Math. Ann. 326 (2003), no. 4, 759-801.

[OS] P. Ozsváth and Z. Szabó, On knot Floer homology and lens space surgeries, Topology 44 (2005), no. 6, 1281-1300.

[Pe] K. Petersen, A-polynomials of a family of two-bridge knots, New York J. Math. 21 (2015), 847881.

[Ri] R. Riley, Nonabelian representations of 2-bridge knot groups, Quart. J. Math. Oxford Ser. (2) 35 (1984), no. 138, 191-208.

[Tr1] A. Tran, Left orderable surgeries of double twist knots, preprint, arXiv:1911.03798.

[Tr2] A. Tran, On left-orderable fundamental groups and Dehn surgeries on knots, J. Math. Soc. Japan 67 (2015), no. 1, 319-338. 
Institute of Mathematics, VAST, 18 Hoang Quoc Viet, 10307, Hanoi, Vietnam

E-mail address: vtkhoi@math.ac.vn

Department of Mathematics and Mathematics Education, Hiroshima University, 1-1-1 Kagamiyama, Higashi-Hiroshima, 739-8524, JaPAN

E-mail address: teragai@hiroshima-u.ac.jp

Department of Mathematical Sciences, The University of Texas at Dallas, RichardSON, TX 75080-3021, USA

E-mail address: att140830@utdallas.edu 